\title{
AN OPTIMIZATION OF THE PHASE-PLANE-DELTA METHOD FOR THE SOLUTION OF NON-LINEAR DIFFERENTIAL EQUATIONS*
}

\author{
BY \\ CARI, A. LUDEKE (University of Cincinnati) \\ AND \\ RICHARD R. WEBER (The Cincinnati Milling Machine Co.)
}

\begin{abstract}
An improvement to the phase-plane-data method of solving non-linear differential equations of the type $d^{2} x / d t^{2}+H(x)=0$ is discussed. This improvement provides a means of determining an optimized value of the parameter $p$, frequency. In the conventional phase-plane-delta method the parameter $p$ is chosen either arbitrarily or as the coefficient of the positive linear term. The phase-plane trajectory and period of oscillation can be more readily determined by this method than by the conventional phase-plane-delta method.

Introduction. Graphical techniques provide simple and rapid means of evaluating the solutions of certain non-linear differential equations. Depending on the extent to which the techniques are carried these graphical solutions will provide varying degrees of qualitative or quantitative information. The phase-plane-delta method, hereafter referred to simply as the $\delta$-method, as described by Jacobsen [1], is a simple graphical technique with good accuracy characteristics. However, the results obtained by the method theoretically approach those of an exact solution only as the size of the individual steps employed in the solution decreases and consequently the number of such steps increases. This requires additional time and effort thereby removing a degree of simplicity so desirable to graphical techniques. Consequently, in a later discussion [2] of this technique, Jacobsen and Ayre suggest that the use of larger steps would in some cases give results of satisfactory accuracy. It is this point that the authors have herein considered. By allowing the value of frequency, $p$, to change from interval to interval it is possible to introduce an optimization procedure which results in better accuracy for a given number of steps. This optimization procedure also overcomes certain difficulties associated with the $\delta$-method.
\end{abstract}

Discussion of Technique. The $\delta$-method is applicable to non-linear differential equations of the type:

$$
m \frac{d^{2} x}{d t^{2}}+G\left(x, \frac{d x}{d t}, t\right)=0
$$

but the refinement is to be considered only in relation to equations of the type

$$
m \frac{d^{2} x}{d t^{2}}+H(x)=0
$$

Following the usual procedure [2] of the $\delta$-method, a positive linear term in $x$ is either separated out of the function $H(x)$ or added to it to give

$$
m \frac{d^{2} x}{d t^{2}}+k x+h(x)=0 \text {. }
$$

${ }^{*}$ Received July 21, 1961. 
Using the usual phase-plane coordinates $(1 / p d x / d t, x)$ Eq. (3) becomes

$$
\frac{d^{2} x}{d t^{2}}+p^{2}(x+\delta)=0,
$$

where

$$
p^{2}=\frac{k}{m} \quad \text { and } \quad \delta=\frac{1}{k} h(x)
$$

giving the values of $p$ and $\delta$ to be used in the $\delta$-method of graphical solution.

Now recall that the basic assumption of the $\delta$-method is that, for small changes of the variable comprising $\delta$, the value of $\delta$ remains essentially constant. It is evident that, when large steps are used in carrying out the solution, this is not strictly true; but it is more and more satisfied as the size of the steps decreases. Therefore, the authors have established a refinement of this technique; namely, that the change in $\delta$ with respect to the variable $x$ is to be a minimum over the interval of the step. This gives the condition

$$
\frac{d}{d x}(\delta)=0 .
$$

It will be shown, in the following examples, that by using this criterion certain important improvements will arise in the application of the $\delta$-method, namely, (a) the value of $p$ used in the solution will depend not on the coefficient of the linear term alone but will be affected by the form of $h(x)$ and (b) it overcomes the difficulty associated with the choice of a value of $p$ when a positive linear term in $x$ does not occur in the original equation.

Applications. As a general presentation of this idea consider the second order non-linear differential equation in which the restoring force is described by a function $f(x)$ that is specified either graphically or analytically and may or may not contain a linear term in $x$

$$
\frac{d^{2} x}{d t^{2}}+f(x)=0 .
$$

Then following the usual steps in the procedure of the $\delta$-method, it is necessary first to introduce a fictitious linear term in $x$

$$
\frac{d^{2} x}{d t^{2}}+p^{2} x-p^{2} x+f(x)=0
$$

so that

$$
\frac{d^{2} x}{d t^{2}}+p^{2}(x+\delta)=0,
$$

where

$$
\delta=\frac{1}{p^{2}}\left[f(x)-p^{2} x\right] .
$$

Upon applying to Eq. (9) the condition expressed by Eq. (5) we find

$$
\frac{1}{p^{2}} \frac{d}{d x}[f(x)]-1=0 .
$$


Hence giving

$$
p^{2}=\frac{d}{d x}[f(x)]
$$

Next it is desired to obtain average values of $p$ and $\delta$ to be used for a particular step; that is, an interval from $x_{1}$ to $x_{2}$ of the solution. The average value of $p$ is determined

$$
\left(p^{2}\right)_{\mathrm{av}}=\frac{1}{x_{2}-x_{1}} \int_{x_{1}}^{x_{2}}\left(p^{2}\right)_{\mathrm{av}} d x
$$

Substituting Eq. (10)

$$
\left(p^{2}\right)_{\mathrm{av}}=\frac{1}{x_{2}-x_{1}} \int_{x_{1}}^{x_{2}} \frac{d}{d x}[f(x)] d x
$$

gives

$$
\left(p^{2}\right)_{\mathbf{a v}}=\left[f\left(x_{2}\right)-f\left(x_{1}\right)\right] /\left[x_{2}-x_{1}\right] .
$$

Similarly, the average value of $\delta$, using Eq. (9) is

$$
\begin{gathered}
\delta_{\mathrm{av}}=\frac{1}{x_{2}-x_{1}} \int_{x_{1}}^{x_{2}} \frac{1}{\left(p^{2}\right)_{\mathrm{av}}}\left[f(x)-\left(p^{2}\right)_{\mathrm{av}} x\right] d x, \\
\delta_{\mathrm{av}}=\frac{1}{\left(p^{2}\right)_{\mathrm{av}}\left(x_{2}-x_{1}\right)} \int_{x_{1}}^{x_{2}} f(x) d x-\frac{1}{\left(x_{2}-x_{1}\right)} \int_{x_{1}}^{x_{2}} x d x, \\
\delta_{\mathrm{av}}=\frac{1}{\left(p^{2}\right)_{\mathrm{av}}\left(x_{2}-x_{1}\right)} \int_{x_{1}}^{x_{2}} f(x) d x-\frac{1}{2}\left(x_{2}+x_{1}\right) .
\end{gathered}
$$

Using Eqs. (13) and (16) we can calculate the various values of $\delta$ and $p$ required to construct the approximate phase-plane trajectory. It is apparent that if $f(x)$ is given analytically the integral in Eq. (16) can be evaluated analytically or numerically, and if $f(x)$ is given as an experimentally determined relation the integral will need to be evaluated by some graphical procedure. Thus $f(x)$ need not be given in an analytical form.

In order to illustrate the procedure for applying this refinement to the $\delta$-method and to point up the advantages that it provides in the phase-plane solution, let us consider two examples. In each of these examples the results, using the usual procedures of the $\delta$-method, will be compared with results obtained using the refinement. Also, the comparison of solutions will be shown employing one, two and four steps in one quadrant of the phase-plane. Only one quadrant is needed since these examples have been chosen so that their phase-plane trajectories are symmetric about the $x$ and $1 / p(d x / d t)$ axes.

I. Consider:

$$
\frac{d^{2} x}{d t^{2}}+x+4 x^{3}=0
$$

with the initial conditions: at $t=0, x=1.0$

for which, using Eq. (13)

$$
\frac{d x}{d t}=0
$$

$$
p_{\mathrm{av}}^{2}=1+4\left(x_{1}^{2}+x_{1} x_{2}+x_{2}^{2}\right)
$$


and using Eq. (16)

$$
\delta_{\mathrm{av}}=\frac{x_{2}+x_{1}}{2 p_{\mathrm{av}}^{2}}\left[2\left(x_{2}^{2}+x_{1}^{2}\right)+\left(1-p_{\mathrm{av}}^{2}\right)\right] .
$$

For a one-step solution (the number of steps refer to the number in one quadrant of the phase-plane) the interval would be $1.0 \geq x \geq 0.0$ giving

\begin{tabular}{l|l|l} 
Interval & $p_{a v}{ }^{2}$ & $\delta_{a v}$ \\
\hline $1.0 \geq x \geq 0.0$ & 5.0 & -0.20
\end{tabular}

The construction of the approximate phase plane trajectory is shown in Fig. 1 and is

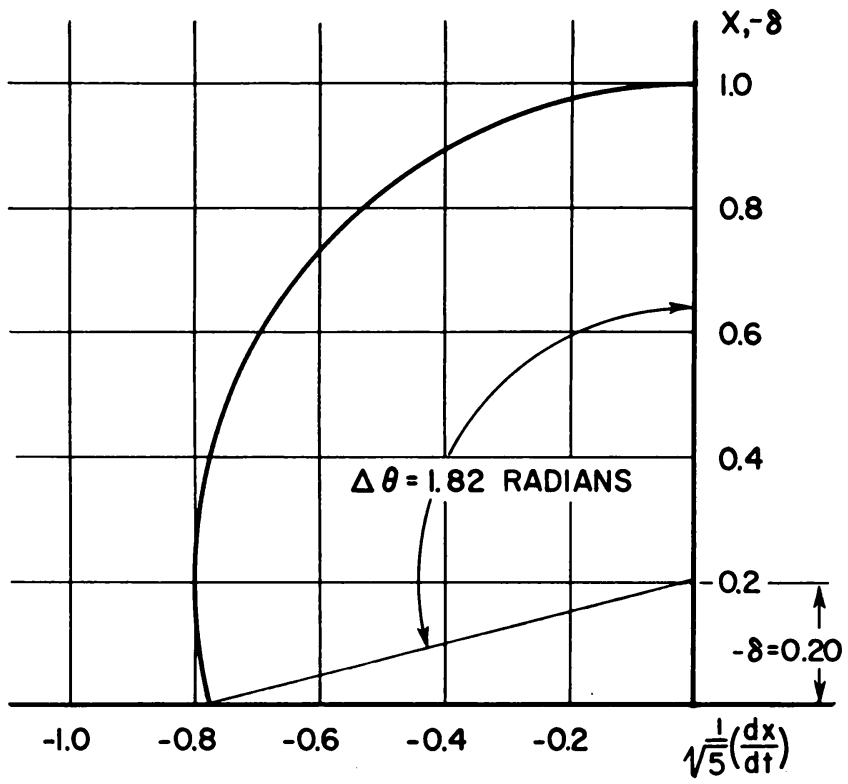

Fig. 1. Construction of $d^{2} x / d t^{2}+x+4 x^{3}=0$ by the optimized $\delta$-method in one-step.

seen to be the same as that for the usual $\delta$-method construction. From which the period for one cycle is, by using the usual expression

$$
\Delta T=\frac{1}{p} \Delta \theta
$$

found to be

$$
T=3.28 \text { seconds }
$$

For a two-step solution

\begin{tabular}{l|c|c} 
Interval & $p_{a v^{2}}$ & $\delta_{a v}$ \\
\hline $1.0 \geq x \geq 0.5$ & 8.0 & -0.42 \\
\hline $0.5 \geq x \geq 0.0$ & 2.0 & -0.06
\end{tabular}




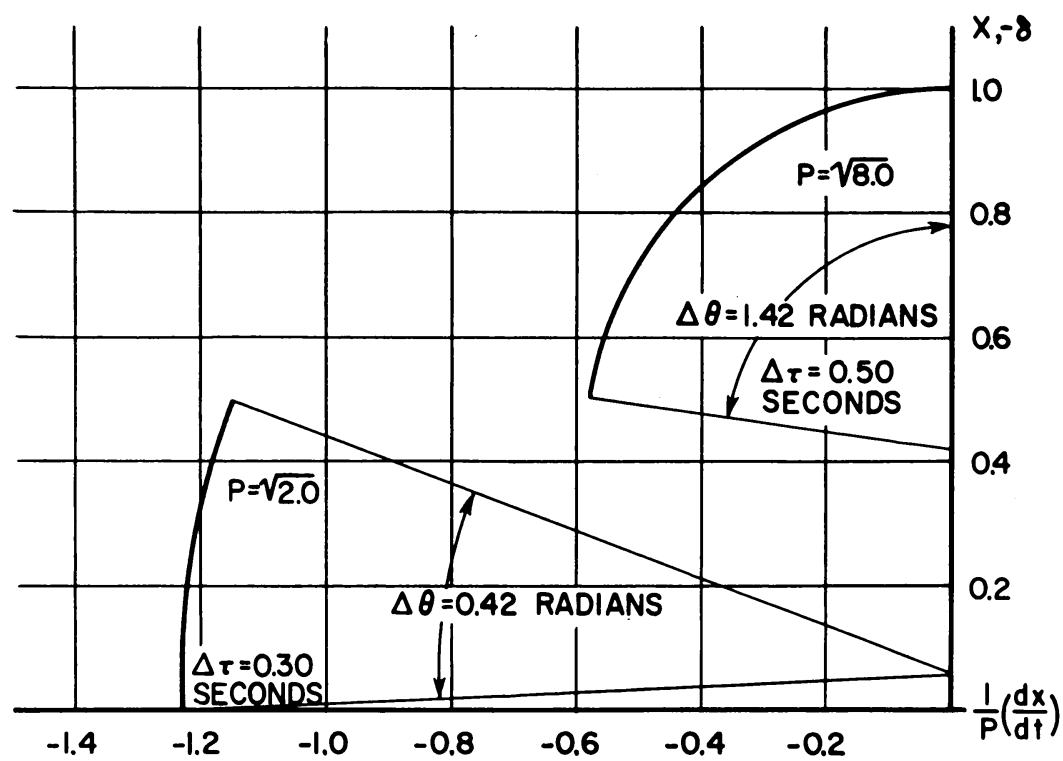

Fig. 2. Construction of $d^{2} x / d t^{2}+x+4 x^{3}=0$ by the optimized $\delta$-method in two steps.

The construction of the approximate phase-plane trajectory is shown in Fig. 2. Here again the construction is the same as that for the usual $\delta$-method construction with the exception that the value of $P_{a v}$ varies from one interval to the next and hence the velocity at the end point of the first interval must be calculated using $p_{a v}=(8.0)^{1 / 2}$ and then replotted as the initial point of the second interval by using $p_{a v}=(2.0)^{1 / 2}$. From which the period for one cycle is found to be

$$
T=3.20 \text { seconds. }
$$

This description of the two constructions along with knowledge of the $\delta$-method is sufficient to describe the optimized procedure; therefore, in the conclusion of this example and the discussion of two other examples only the tables for $p_{\mathrm{av}}^{2}$ and $\delta_{a v}$ along with the resulting period will be included.

For the four step solution

\begin{tabular}{l|c|c} 
Interval & $p_{a v}{ }^{2}$ & $\delta_{a v}$ \\
\hline $1.00 \geq x \geq 0.75$ & 10.25 & -0.52 \\
\hline $0.75 \geq x \geq 0.50$ & 5.75 & -0.34 \\
\hline $0.50 \geq x \geq 0.25$ & 2.75 & -0.15 \\
\hline $0.25 \geq x \geq 0.00$ & 1.25 & -0.01
\end{tabular}

and the period determined from the construction is found to be

$$
T=3.18 \text { seconds. }
$$


A numerical solution of the phase-plane trajectory can be obtained by integrating this non-linear differential equation once. Also the exact period can be calculated from an elliptic integral to be

$$
T=3.18 \text { seconds. }
$$

Table I below compares the results of the periods obtained by this optimized technique with the usual technique which would use $p^{2}=1.0$ in this particular case, since $p^{2}$ would be understood to be the coefficient of the linear term in $x$. Figure 3 shows a com-

TABLE 1

Comparison of the Optimized Procedure and Conventional Procedure of the $\delta$-Method for $d^{2} x / d t^{2}+x+4 x^{3}=0$

\begin{tabular}{|c|c|c|c|c|c|}
\hline \multirow{2}{*}{$\begin{array}{l}\text { Number of } \\
\text { Steps in One } \\
\text { Quadrant }\end{array}$} & \multicolumn{5}{|c|}{ Period (seconds) } \\
\hline & Exact & Conventional & $\begin{array}{c}\% \\
\text { Error }\end{array}$ & Optimized & $\begin{array}{c}\% \\
\text { Error }\end{array}$ \\
\hline 1 & 3.18 & 4.19 & 32 & 3.28 & 3 \\
\hline 2 & & 3.53 & 11 & 3.20 & 1 \\
\hline 4 & & 3.14 & 1 & 3.18 & - \\
\hline
\end{tabular}

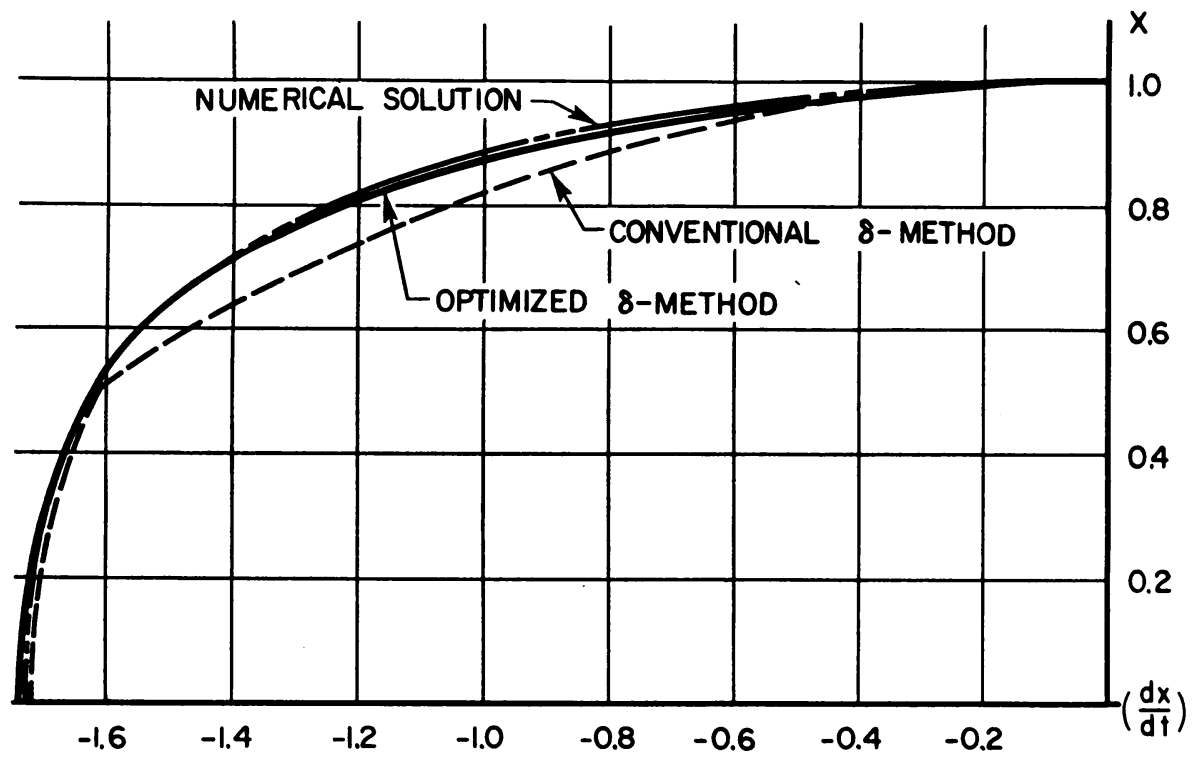

FIg. 3. Phase-plane trajectory of $d^{2} x / d t^{2}+x+4 x^{3}=0$.

parison of the phase-plane trajectories determined by these two techniques with the numerically determined trajectory. Two steps were used in the graphical solutions. II. Consider

$$
\frac{d^{2} x}{d t^{2}}+x^{3}=0
$$

with the initial conditions: at $t=0, x=2.0, d x / d t=0.0$ which represents the case of a 
non-linear differential equation where the positive linear term is missing and hence the value of $p^{2}$, for the conventional $\delta$-method, cannot be determined but would have to be estimated. The procedure of the optimized technique, on the other hand, gives a direct method of determining this value by using Eq. (13)

and using Eq. (16)

$$
p_{\mathrm{av}}^{2}=\left[x_{2}^{3}-x_{1}^{3}\right] /\left[x_{2}-x_{1}\right]
$$

$$
\delta_{\mathrm{av}}=\left(x_{2}+x_{1}\right)\left[\left(x_{2}^{2}+x_{1}^{2}\right) / 4 p_{\mathrm{av}}^{2}-\frac{1}{2}\right] .
$$

The values required for the construction of the phase-plane trajectories of this equation are given below:

\begin{tabular}{l|c|c|c} 
& Interval & $p_{a v}{ }^{2}$ & $\delta_{a v}$ \\
\hline \hline $\begin{array}{l}\text { One Step } \\
\text { Solution }\end{array}$ & $2.0 \geq x \geq 0.0$ & 4.0 & -0.50 \\
\hline Two Step & $2.0 \geq x \geq 1.0$ & 7.0 & -0.96 \\
Solution & $1.0 \geq x \geq 0.0$ & 1.0 & -0.25 \\
\hline & $2.0 \geq x \geq 1.5$ & 9.25 & -1.16 \\
& $1.5 \geq x \geq 1.0$ & 4.75 & -0.82 \\
Four Step & $1.0 \geq x \geq 0.5$ & 1.75 & -0.48 \\
Solution & $0.5 \geq x \geq 0.0$ & 0.25 & -0.13 \\
& &
\end{tabular}

The $\delta$-method graphical solutions, constructed using the values above, are compared in Table II below with values obtained from a solution assuming $p^{2}=1$, and with an exact numerical solution. Figure 4 shows a comparison of the phase-plane trajectories determined by these two techniques with the numerically determined trajectory. Once again, two-steps are used in the graphical solutions.

TABLE II

Comparison of the Optimized Procedure and Conventional Procedure

\begin{tabular}{|c|c|c|c|c|c|}
\hline \multirow{2}{*}{$\begin{array}{l}\text { Number of } \\
\text { Steps in One } \\
\text { Quadrant }\end{array}$} & \multicolumn{5}{|c|}{ Period (seconds) } \\
\hline & Exact & Conventional & $\begin{array}{c}\% \\
\text { Error }\end{array}$ & Optimized & $\begin{array}{c}\% \\
\text { Error }\end{array}$ \\
\hline 1 & 3.71 & 4.88 & 32 & 3.82 & 3 \\
\hline 2 & & 4.19 & 13 & 3.74 & 1 \\
\hline 4 & & 3.81 & 3 & 3.72 & - \\
\hline
\end{tabular}
of the $\delta$-Method for $d^{2} x / d t^{2}+x^{3}=0$

In both of the examples illustrated above, the form of $f(x)$ was given analytically. However, it is obvious that this need not be the case and that $f(x)$ can be described graphically. Such an example has not been included as its discussion would not introduce any new facets of the technique and furthermore could not be solved analytically to show a comparison of results as was done for the examples included. However, the authors have applied the optimized $\delta$-method to such problems and found the technique to be very advantageous in that it gave a faster convergence to the period. 


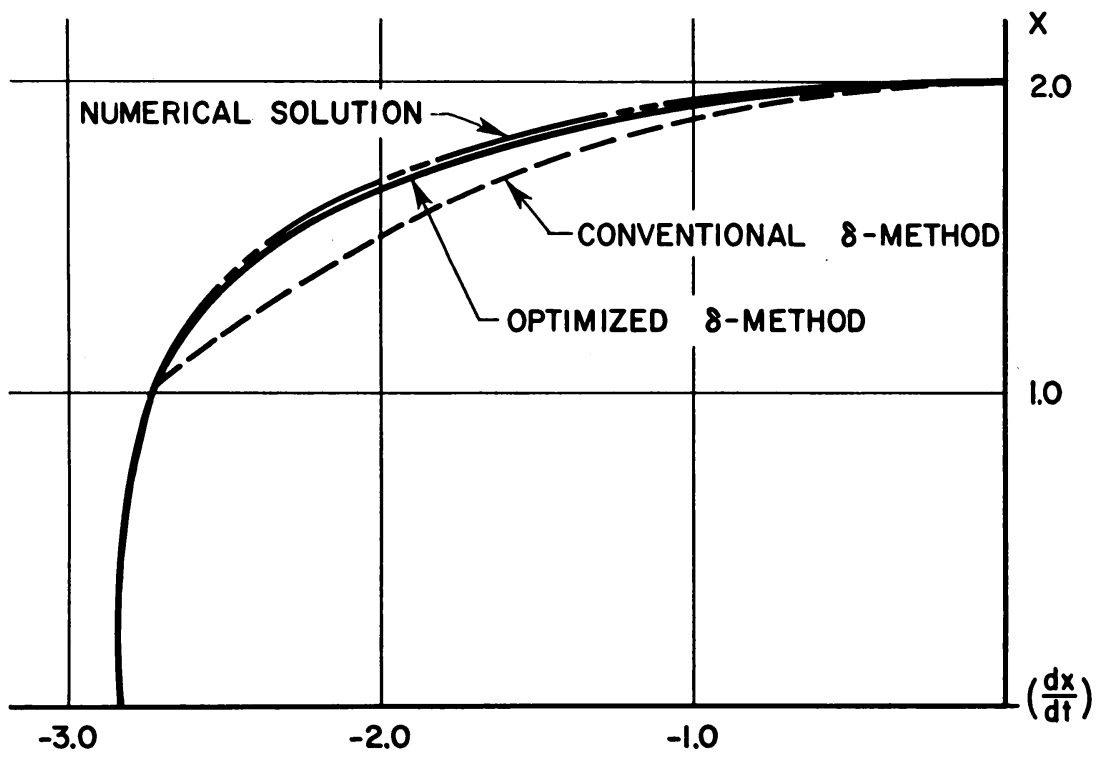

Fig. 4. Phase-plane trajectory of $d^{2} x / d t^{2}+x^{3}=0$.

Discussion of convergence. The convergence of the period to the exact period is seen in the cases illustrated to be significantly more rapid when the optimized $\delta$-method is used. However, discussion of this result can only be empirical and drawn from the authors' experiences with several examples. From these results it appears that this rate of convergence is related to the area between the actual curve of the function $f(x)$ and the approximating straight line segments determined by $p_{\mathrm{av}}^{2}$. Thus, it is always desirable to distribute the number of segments into which the solution is to be divided so that the area between the straight line approximation and the actual function $f(x)$ is a minimum. The area which is to be minimized is shown shaded in Fig. 5. It will be noted, recalling Eq. (13), that the slopes of these line segments are then $p_{\mathrm{av}}^{2}$ for a particular interval. As further illustration of this point, this last example is included in which the

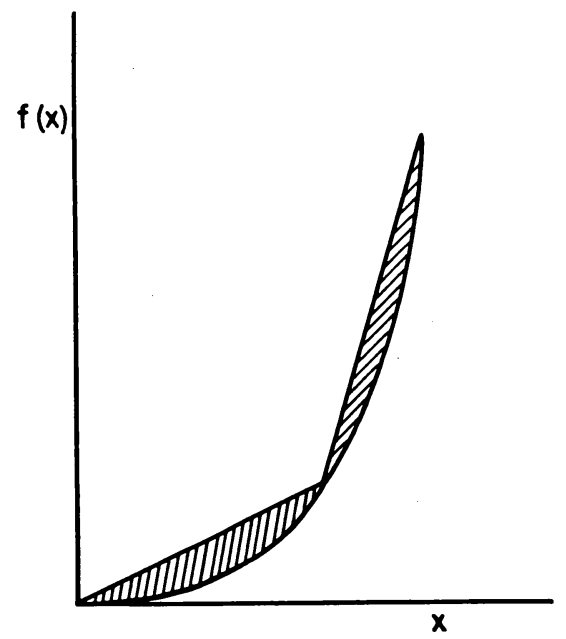

FIG. 5. Diagram showing the criterion for the choice of the straight-line approximations. 
choice of intervals is taken so as to reduce continuously the area between the approximating straight-line segments and the actual function curve.

Consider

$$
\frac{d^{2} x}{d t^{2}}+f(x)=0
$$

with the initial conditions at $t=0, x=2.5, d x / d t=0.0$ and where

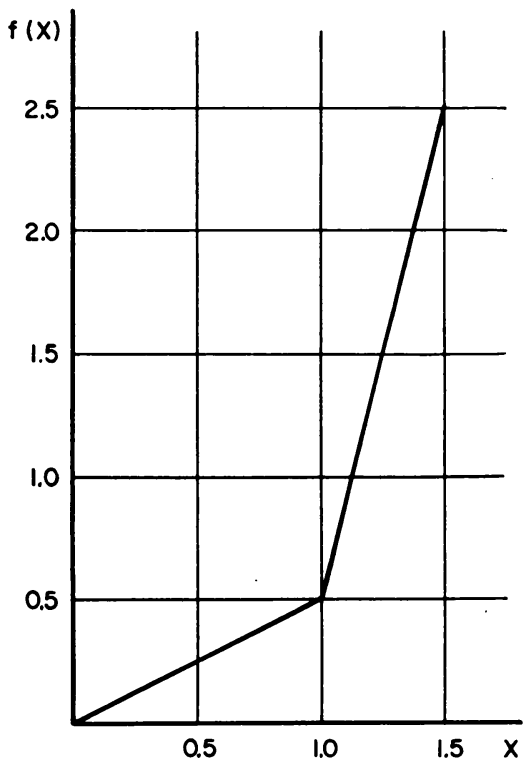

In Table III below are shown five cases involving successively improving approximations TABLE III

Error Obtained for Successively Improving Approximations to the Actual Restoring Force Function $f(x)$

\begin{tabular}{|c|c|c|c|c|c|}
\hline \multirow[b]{2}{*}{ Case } & \multirow[b]{2}{*}{ Interval } & \multicolumn{3}{|c|}{ Period (seconds) } & \multirow[b]{2}{*}{$\Delta A / A$} \\
\hline & & Exact & Optimized & $\begin{array}{c}\% \\
\text { Error }\end{array}$ & \\
\hline 1 & $1.5 \geq x \geq 0.0$ & 1.425 & 1.460 & 2.4 & 0.875 \\
\hline 2 & $\begin{array}{l}1.5 \geq x \geq 1.375 \\
1.375 \geq x \geq 0.0\end{array}$ & & 1.442 & 1.2 & 0.656 \\
\hline 3 & $\begin{array}{l}1.5 \geq x \geq 1.25 \\
1.25 \geq x \geq 0.0\end{array}$ & & 1.404 & 1.5 & 0.438 \\
\hline 4 & $\begin{array}{l}1.5 \geq x \geq 1.125 \\
1.125 \geq x \geq 0.0\end{array}$ & & 1.415 & 0.7 & 0.219 \\
\hline 5 & $\begin{array}{l}1.5 \geq x \geq 1.0 \\
1.0 \geq x \geq 0.0\end{array}$ & & 1.425 & - & 0.000 \\
\hline
\end{tabular}


to the restoring force function, $f(x)$. In this table is also shown the ratio of the difference between the area under the curve, consisting of the approximating straight-line segments, and the area under the curve of the actual restoring force to the area under the curve of the actual restoring force. This is designated as $\Delta A / A$. Similar summaries are shown in Tables IV and V below for the two examples previously discussed. From the results

TABLE IV

Error and Values of $\Delta A / A$ for the Various Approximations

of the Optimized $\delta$-Method for $d^{2} x / d t^{2}+x+4 x^{3}=0$

\begin{tabular}{|c|c|c|c|c|c|}
\hline \multirow[b]{2}{*}{ Case } & \multirow[b]{2}{*}{ Interval } & \multicolumn{3}{|c|}{ Period (seconds) } & \multirow[b]{2}{*}{$\Delta / A$} \\
\hline & & Exact & Optimized & $\begin{array}{c}\% \\
\text { Error }\end{array}$ & \\
\hline 1 & $1.0 \geq x \geq 0.0$ & 3.18 & 3.28 & 3 & 0.67 \\
\hline 2 & $\begin{array}{l}1.0 \geq x \geq 0.5 \\
0.5 \geq x \geq 0.0\end{array}$ & & 3.20 & 0.6 & 0.17 \\
\hline 3 & $\begin{array}{l}1.0 \geq x \geq 0.75 \\
0.75 \geq x \geq 0.50 \\
0.50 \geq x \geq 0.25 \\
0.25 \geq x \geq 0.00\end{array}$ & & 3.18 & - & 0.04 \\
\hline
\end{tabular}

TABLE V

Error and Values of $\Delta A / A$ for the Various Approximations of the Optimized $\delta$-Method for $d^{2} x / d t^{2}+x^{3}=0$

\begin{tabular}{|c|c|c|c|c|c|}
\hline \multirow[b]{2}{*}{ Case } & \multirow[b]{2}{*}{ Interval } & \multicolumn{3}{|c|}{ Period (seconds) } & \multirow[b]{2}{*}{$\Delta A / A$} \\
\hline & & Exact & Optimized & $\begin{array}{c}\% \\
\text { Error }\end{array}$ & \\
\hline 1 & $2.0 \geq x \geq 0.0$ & 3.71 & 3.82 & 3 & 1.00 \\
\hline 2 & $\begin{array}{l}2.0 \geq x \geq 1.0 \\
1.0 \geq x \geq 0.0\end{array}$ & & 3.74 & 0.8 & 0.25 \\
\hline 3 & $\begin{array}{l}2.0 \geq x \geq 1.5 \\
1.5 \geq x \geq 1.0 \\
1.0 \geq x \geq 0.5 \\
0.5 \geq x \geq 0.0\end{array}$ & & 3.72 & - & 0.06 \\
\hline
\end{tabular}

shown in these tables it is seen that when the straight line segments approximate the curve so that $\Delta A / A$ is $25-30 \%$, the error experienced in the period is less than $1 \%$.

Conclusions. The examples shown are typical of the experiences of the authors in applying this optimized $\delta$-method to the graphical solution and has been found to have the following advantages. 
1. The period calculated using the optimized $\delta$-method results in a value better approximating the exact value of the period than the conventional $\delta$-method.

2 . In those cases where there is not a linear term in the non-linear differential equation to give a value of $p_{\mathrm{av}}^{2}$ to be used in the conventional $\delta$-method or where the magnitude of the non-linearity is of such a size as to make the use of the frequency as specified by the linear term unrealistic the optimized $\delta$-method provides a direct method of calculating an appropriate value of $p_{\mathrm{av}}^{2}$.

3. The accuracy that can be obtained for the period with the optimized $\delta$-method has been empirically found to be better than $1 \%$ when the straight line segments approximate the actual restoring force function to within a value of $25-30 \%$, for the ratio of $\Delta A / A$.

4. The authors also have observed that the phase-plane trajectories determined by the optimized $\delta$-method have less distortion from the actual trajectory than those determined by the conventional $\delta$-method.

5. Finally, a graphical technique to be useful should also be relatively simple. The experience of the authors again in this instance has been that no appreciable complexity is introduced by the precalculation that must be performed and in fact this is far offset by the reduction in the number of steps required to determine the period and phaseplane trajectory to some particular degree of accuracy.

\section{BIBLIOGRAPHY}

1. L. S. Jacobsen, On a general method of solving second-order ordinary differential equations by phase-plane displacements, J. Appl. Mechanics 19, 543-553 (1952)

2. L. S. Jacobsen and R. S. Ayre, Engineering vibrations, McGraw-Hill Book Co., Inc., New York, N. Y., 1958, pp. 244-259 